\title{
Prevalence of and factors associated with expressed and unmet service needs reported by persons with spinal cord injury living in the community
}

\author{
Bruno Trezzini ${ }^{1,2} \cdot$ Mirjam Brach $^{1,2} \cdot$ Marcel Post $^{1,3,4} \cdot$ Armin Gemperli $^{1,2} \cdot$ for the SwiSCI Study Group
}

Received: 9 September 2018 / Revised: 11 January 2019 / Accepted: 11 January 2019 / Published online: 29 January 2019

(c) International Spinal Cord Society 2019

\begin{abstract}
Study design Observational study.

Objectives To determine the prevalence, predictors, and consequences of self-reported service needs among communitydwelling persons with SCI.

Setting Community-based, Switzerland.

Methods Participants were 490 people who took part in the health services module of the Swiss Spinal Cord Injury Cohort Study (SwiSCI) 2012 community survey. We determined the prevalence of 20 service needs and the extent to which they were unmet. Predictors and consequences of service needs were examined with multiple regression analyses.

Results High-prevalence needs (e.g., general health care, accessible housing) exhibited a high level of fulfillment. Conversely, less prevalent service needs (e.g., peer support, support for family caregivers) showed lower levels of fulfillment. Across three specific service domains (peer support, support for family caregivers, sports activities), lower household income predicted most consistently a higher likelihood of unmet needs. The total number of expressed needs was higher in non-Swiss nationals, persons with complete para- or tetraplegia and lower income individuals. Being female, French language region and lower household income predicted more total unmet needs. Increased expressed and unmet service needs were associated with lower life satisfaction.

Conclusions Service needs with a high prevalence seem to be adequately met by the current service provision system. However, rehabilitation professionals should remain alert to clients' specific and cumulative unmet needs, in particular with respect to less common ones, and their impact on successful community reintegration and life satisfaction.
\end{abstract}

Supplementary information The online version of this article (https:// doi.org/10.1038/s41393-019-0243-y) contains supplementary material, which is available to authorized users.

$\triangle$ Bruno Trezzini

bruno.trezzini@paraplegie.ch

1 Swiss Paraplegic Research, Nottwil, Switzerland

2 Department of Health Sciences and Health Policy, University of Lucerne, Lucerne, Switzerland

3 Center of Excellence for Rehabilitation Medicine, Rudolf Magnus Institute of Neuroscience, University Medical Center Utrecht and De Hoogstraat, Utrecht, The Netherlands

4 University of Groningen, University Medical Center Groningen, Department of Rehabilitation Medicine, Groningen, The Netherlands

\section{Introduction}

In order to fully participate in society on an equal basis with others and to achieve and maintain high levels of wellbeing, persons with a disability require various kinds of assistance and support to address their health conditionrelated needs in different areas of life [1, 2]. For persons with spinal cord injury (SCI), previous studies have identified a broad array of health condition-related needs [3-5]. However, even in countries with generally high service provision levels and individualized care, unmet needs in domains such as attendant care, accessible housing, assistive devices, transportation, leisure activities or sexuality were found to be relatively prevalent [6-14].

Several studies investigated satisfaction with available services [9, 12, 15], main providers of particular services [12], preferred channels of service provision [6], barriers to needs being met $[6,12]$, and impact on quality of life 
$[9,16]$. For example, in a Dutch study $45 \%$ of respondents gave a score of 4.5 or more out of 5 with regard to their general satisfaction with available services, regardless of type of injury, age, education, and income [9]. By contrast, levels of satisfaction with service delivery procedures turned out to be lower, especially in persons with a complete as compared to an incomplete injury. In terms of barriers to needs being met, Cox et al. [6] identified limited local SCI-specialized knowledge and inadequate funding as major obstacles in an Australian sample, and a majority of the Canadian participants in Noreau et al. [12] completely disagreed with the statement that the process of getting one's needs met regarding accessible housing (64\%) and income support $(50 \%)$ was easy. With regard to potential consequences of unmet needs and gaps in health care and social service provision, Sweet et al. [16] found a direct positive association with the frequency of secondary health conditions, such as bowel-bladder complications and pressure ulcers, and a direct negative association with quality of life.

It warrants highlighting that comparisons of results across studies are often hampered owing to differences in how the service need information was elicited and how gaps in the fulfillment of such needs were operationalized. Furthermore, to address the shortage of evidence documenting service needs, and whether they are being met, more largescale, nation-wide surveys such as presented in Noreau et al. [13] are needed. Even in the case of a high-resource country such as Switzerland there are currently no reliable data available on the type and prevalence of service needs among community-dwelling persons with SCI. A crossnational study involving four European countries included merely 27 participants from Switzerland and did not report results for individual countries [17].

The objective of this study was to investigate how well existing support service systems in a highly developed country such as Switzerland meet perceived needs after rehabilitation among people with SCI and what the associated antecedents and consequences of potential gaps are. By broadening the empirical evidence base, the study intended to provide a solid foundation for further improving current service provision systems. More specifically, the aims of this study were:

(1) to assess the relationship between prevalence and level of fulfillment of specific service needs of community-dwelling persons with SCI;

(2) to determine sociodemographic and injury-related predictors of expressed and unmet service needs, both for selected specific and aggregated needs;

(3) to determine the association of total number of expressed and unmet service needs with life satisfaction adjusted for sociodemographic and injury-related covariates.

\section{Methods}

\section{Study design}

We used cross-sectional observational data of communitydwelling participants.

\section{Participants}

The study sample originated from the 2012 community survey conducted as part of the Swiss Spinal Cord Injury Cohort Study (SwiSCI) [18]. The SwiSCI target population comprised all persons age 16 or older, with a traumatic or non-traumatic SCI diagnosis and permanent Swiss residency [18]. Persons with congenital conditions, neurodegenerative disorders and Guillain-Barré syndrome were excluded. The sampling frame consisted of records from specialized SCI rehabilitation clinics and patient organizations. The initial eligible population comprised 3144 persons. Of the 1549 SwiSCI participants who had completed an earlier questionnaire covering functioning, health conditions, environmental and personal factors, 580 randomly selected persons were invited to answer additional questions related to health services.

\section{Variables}

\section{Service needs}

Drawing on previous research [9-11, 13], we assessed perceived needs as dependent variables (aim 1 and 2) by means of 20 items covering domains such as "accessible housing", "SCI-specialized health care", "peer support" or "support for family caregivers". After clarifying that "coverage of a need" should be interpreted as "the actual need" and not "a perceived legal entitlement", and that service provision could involve both private and government means, participants were asked: "How well is your current need covered in the following life areas?'. The available answer options were "not at all", "to a small extent", "to some extent", "to a large extent", "completely", and "I don't have such a need". The obtained information on service needs was further processed as follows. First, we created a variable indicating the degree of need fulfillment by assigning the value range $0-4$ to the above first five answer categories. Second, we created two dichotomous variables that indicated (a) whether a respondent had a specific need or not (expressed need), and if so, (b) whether the need was met or not (unmet need). The answer categories "not at all", "to a small extent", and "to some extent" were considered as indicating the existence of an unmet need. The expressed need information was used to determine need prevalence $(0-100 \%)$. Third, based on these two dichotomized variables, we derived two additional 
Table 1 Sample characteristics $(n=490)$

\begin{tabular}{|c|c|c|}
\hline Categorical variables $[n$ missing] & $n$ & $\%$ \\
\hline \multicolumn{3}{|l|}{ Gender [0] } \\
\hline Male & 348 & 71.0 \\
\hline Female & 142 & 29.0 \\
\hline \multicolumn{3}{|l|}{ Living alone [10] } \\
\hline No & 367 & 76.5 \\
\hline Yes & 113 & 23.5 \\
\hline \multicolumn{3}{|l|}{ Language region $[0]$} \\
\hline German & 342 & 69.8 \\
\hline French & 125 & 25.5 \\
\hline Italian & 23 & 4.7 \\
\hline \multicolumn{3}{|l|}{ Nationality [11] } \\
\hline Swiss & 441 & 92.1 \\
\hline Non-Swiss & 38 & 7.9 \\
\hline \multicolumn{3}{|l|}{ Household income [24] } \\
\hline$<$ CHF 1500 & 11 & 2.4 \\
\hline CHF $1500-3000$ & 37 & 7.9 \\
\hline CHF $3000-4500$ & 86 & 18.5 \\
\hline CHF $4500-6000$ & 134 & 28.8 \\
\hline CHF $6000-7500$ & 67 & 14.4 \\
\hline CHF 7500-9000 & 63 & 13.5 \\
\hline$>$ CHF 9000 & 68 & 14.6 \\
\hline \multicolumn{3}{|l|}{ Currently employed [1] ${ }^{\mathrm{a}}$} \\
\hline No & 137 & 42.8 \\
\hline Yes & 183 & 57.2 \\
\hline \multicolumn{3}{|l|}{ Type of SCI cause [5] } \\
\hline Non-traumatic & 113 & 23.3 \\
\hline Traumatic & 372 & 76.7 \\
\hline \multicolumn{3}{|l|}{ Injury severity [4] } \\
\hline Paraplegia, incomplete & 190 & 39.1 \\
\hline Paraplegia, complete & 147 & 30.2 \\
\hline Tetraplegia, incomplete & 105 & 21.6 \\
\hline Tetraplegia, complete & 44 & 9.1 \\
\hline Continuous variables $[n$ missing] & Mean & $\mathrm{SD}$ \\
\hline Age at time of survey (years) [0] & 55.4 & 15.1 \\
\hline Age at SCI onset (years) [12] & 38.7 & 18.2 \\
\hline Time since SCI (years) [12] & 16.5 & 13.1 \\
\hline Education (years) [9] & 13.4 & 3.1 \\
\hline
\end{tabular}

${ }^{a}$ Only persons of working age included

variables indicating the cumulative presence of service needs, i.e., the total number of expressed and the total number of unmet needs of a respondent (range 0-20). In deriving the total number of expressed service needs we assumed that if a person did not provide any information on a specific need this may reasonably be interpreted as an indication that the particular need was not present. These variables served as both dependent (aim 2) and independent (aim 3) variables.

\section{Sociodemographic and injury-related factors}

We included gender, age at the time of the survey, nationality (Swiss vs. non-Swiss), education (years), current living arrangement (alone vs. not alone), household income (a seven-point scale ranging from < CHF 1500 to $>$ CHF 9000 treated as an interval-scaled variable), and questionnaire language (German, French, Italian) as a proxy for geographical location within Switzerland as potential sociodemographic explanatory variables for expressed and unmet needs. Injury-related factors included time since SCI onset, injury severity (incomplete paraplegia, complete paraplegia, incomplete tetraplegia, complete tetraplegia) and cause of SCI (traumatic vs. non-traumatic).

\section{Life satisfaction}

Life satisfaction as an outcome variable (aim 2) was evaluated by asking participants the following question: "In general, how satisfied are you with your life if 0 means 'not at all satisfied' and 10 means 'completely satisfied'?" [19].

\section{Data analysis}

Statistical analyses were performed using Stata/MP 14.1 for Windows and R 3.2.1. A descriptive analysis of participants' characteristics and of the prevalence and fulfillment levels of their service needs was conducted. For prevalence data, the confidence intervals were calculated with the logittransform method [20]. Further, we plotted service need prevalence (range 0-100\%) against average degree of need fulfillment (range 0 [not at all] to 4 [completely]) and marked the respective means in the scatterplot, thus defining four quadrants. The south-west and the south-east quadrants are of particular interest because they represent service needs with below average fulfillment levels and, in the latter case, with an above average prevalence. For the two workrelated service needs only respondents of employable age were included in the analysis. The association between prevalence and fulfillment levels was assessed by means of Spearman's rho.

We used fixed effects multiple regression models to examine potential antecedents and consequences of service needs, entering all variables in one step. To evaluate predictors for specific expressed and unmet service needs, we applied logistic regression. To account for potential overdispersion of the count data, total number of expressed and total number of unmet needs were analyzed with negative binomial regressions [21], yielding adjusted incidence rate ratios for the sociodemographic and injury-related predictors. The adjusted effects of total expressed and of total unmet service needs on life satisfaction were assessed by means of linear regression. Visual inspection indicated that 


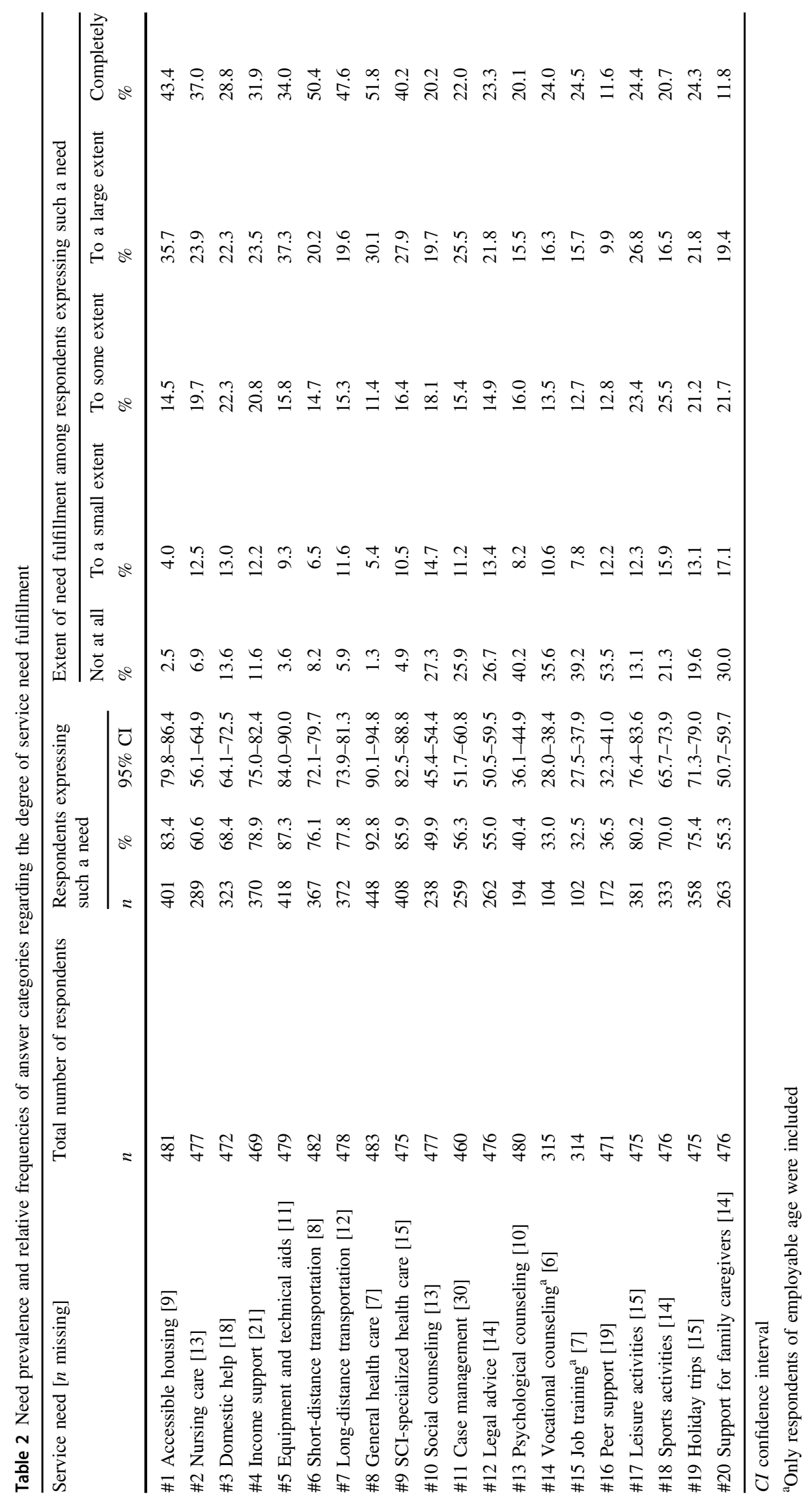




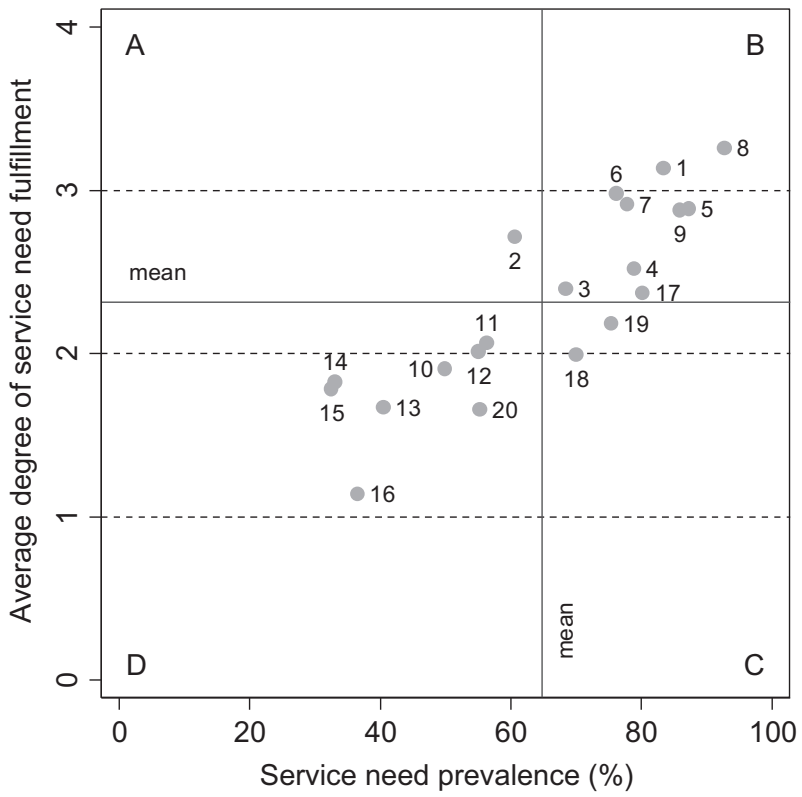

Fig. 1 Scatterplot of degree of service need fulfillment against service need prevalence. Key: \#1 accessible housing, \#2 nursing care, \#3 domestic help, \#4 income support, \#5 equipment and technical aids, \#6 short-distance transportation, \#7 long-distance transportation, \#8 general health care, \#9 SCI-specialized health care, \#10 social counseling, \#11 case management, \#12 legal advice, \#13 psychological counseling, \#14 vocational counseling, \#15 job training, \#16 peer support, \#17 leisure activities, \#18 sports activities, \#19 holiday trips, \#20 support for family caregivers. Reading example: $36.5 \%$ of participants indicated a need for \#16 peer support and their average level of need fulfillment was 1.14 (with $0=$ not at all, $1=$ to a small extent, 2 $=$ to some extent, $3=$ to a large extent, $4=$ completely). Comments: For \#14 vocational counseling and \#15 job training only respondents of employable age were included

after taking squares to address the left skewness of the dependent variable, the linear regression model assumptions of linearity and normality of the residuals were met. Calculated variance inflation factors suggested absence of multicollinearity. Robust standard errors were applied in order to address potential issues of heteroscedasticity.

Missing data were handled as follows. To account for unit nonresponse, we used sampling weights in all regression analyses, calculated as the inverse of the propensity score of participation in the survey module focusing on health services [18]. The mean-standardized sampling weights ranged from 0.4 to 2.0 , suggesting no undue influence of individual participants [18]. We addressed item nonresponse by imputing missing values in the covariates, using a random forest technique implemented in $\mathrm{R}$ [22].

\section{Results}

Of the 492 persons who eventually participated, two respondents with missing data on all service needs items were excluded, leaving the present study sample of 490 participants.

\section{Respondent characteristics}

Table 1 provides an overview of selected sociodemographic and injury-related characteristics of the study participants. Overall, participants were predominantly male $(71.0 \%)$, had an average age of 55.4 years, with 13.4 years of formal education. In terms of household income, $2.4 \%$ fell into the lowest, $28.8 \%$ in the middle, and $14.6 \%$ in the highest income group. Of those respondents who were of employable age at the time of the survey, $57.2 \%$ were gainfully employed. A majority of the respondents lived with paraplegia (69.3\%), $76.7 \%$ had sustained a traumatic SCI and mean time since injury was 16.5 years.

\section{Prevalence and level of fulfillment of service needs}

With regard to the prevalence and fulfillment level of specific service needs, Table 2 and Fig. 1 indicate that general health care (\#8: 92.8\%), equipment and technical aids (\#5: 87.3\%) as well as SCI-specialized health care (\#9: 85.9\%) were the three most prevalent needs. By contrast, the three least prevalent ones were job training (\#15: 32.5\%), vocational counseling (\#14: 33.0\%) and peer support (\#16: $36.5 \%)$. In terms of service need fulfillment, the highest average scores were for general health care (\#8: 3.3), accessible housing (\#1: 3.1) and short-distance transportation (\#6: 3.0). The lowest scores emerged for peer support (\#16: 1.1), support for family caregivers (\#20: 1.7) and psychological counseling (\#13: 1.7). Figure 1 also shows that most service needs fell either into quadrant B or D, only two service needs (sports activities, holiday trips) fell into quadrant $\mathrm{C}$. This was reflected in a statistically significant positive correlation between service need prevalence and average fulfillment level $(\mathrm{n}=20$, rho $=0.859, \mathrm{p}<0.001)$.

Figure 2 shows the frequency distributions of the total number of expressed (right panel) and the total number of unmet (left panel) service needs reported by participants. Eleven persons indicated no service needs whatsoever, whereas 39 indicated that they had needs in all 20 covered areas. The average number of expressed needs was 12.4 (median: 13). With regard to the total number of unmet needs (after dichotomization), 93 persons indicated no unmet service needs at all, whereas one person reported having unmet needs in all 20 areas. The average number of unmet needs was 5.5 (median: 4).

\section{Predictors of expressed and of unmet service needs}

Drawing on the results presented in Fig. 1 and focusing on needs with below average fulfillment levels and 
Fig. 2 Frequency distribution of the total number of expressed and unmet service needs reported by respondents $(n=490)$

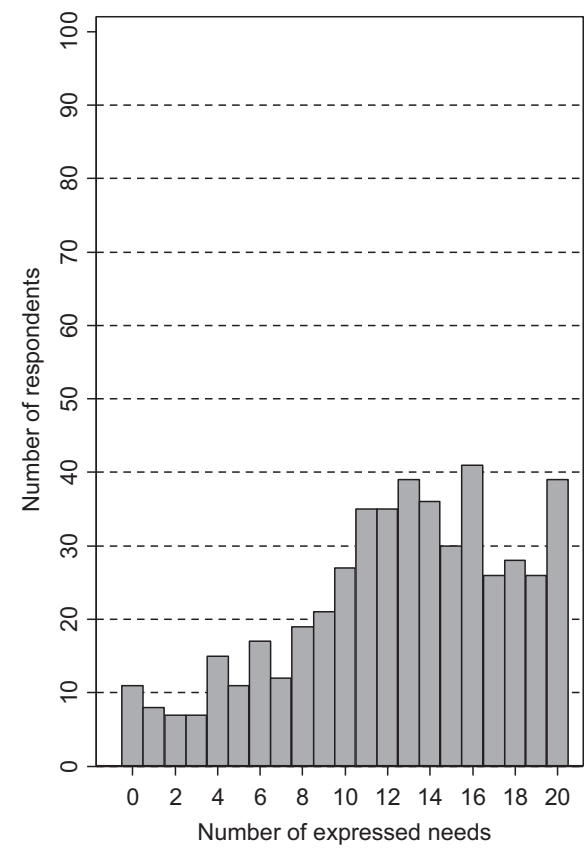

comparatively high prevalence, we selected the three specific areas of \#16 peer support, \#20 support for family caregivers, and \#18 sports activities for further analysis. For a combined view across all 20 domains, we investigated predictors of total number of expressed needs and of total number of unmet needs.

\section{Expressed service needs}

French and Italian language region as well as non-Swiss citizenship were associated with a higher expressed need for peer support and living alone with a lower need (model 1 in Table 3). An expressed need for support for family caregivers (model 2) was positively associated with French language region, non-Swiss citizenship and with incomplete or complete tetraplegia; negative associations were found for household income and a traumatic SCI etiology. The odds for expressing a need related to sports activities (model 3 ) were reduced for older respondents and for longer time since SCI onset. With regard to the total number of expressed needs (model 4), having non-Swiss citizenship and complete paraplegia or complete tetraplegia were positively and household income negatively associated. All models were statistically significant, with pseudo- $R^{2}$ ranging from 0.02 (model 4) to 0.10 (model 1).

\section{Unmet service needs}

Being female, age at time of survey and years of education were positively associated with having an unfulfilled need for peer support (see model 1 in Table 4), whereas household income showed a negative association. An unmet need regarding support for family caregivers (model 2) was positively associated with education years and negatively with household income. Female gender, higher age at time of survey and French language region predicted a higher likelihood for an unmet need for sports activities (model 3), whereas household income and time since SCI onset exhibited a negative association. With regard to the total number of concurrent unmet needs (model 4), having a French or Italian language background, female gender and non-Swiss citizenship were positively associated, whereas household income and time since SCI showed a negative impact. All models were statistically significant, with pseudo- $R^{2}$ ranging from 0.02 (model 4) to 0.28 (model 1).

\section{Impact of total number of expressed and unmet service needs on life satisfaction}

With regard to the potential impact that an accumulation of service needs might have on life satisfaction, we found that the effect of total number of expressed needs on life satisfaction squared was negative (coeff.(b) $=-1.54 ; p<0.001$; 95\% CI: $-2.02,-1.05$ ) (see supplementary Table S1). Of the other socio-demographic and injury-related predictors included in the regression, only non-Swiss citizenship had a statistically significant negative and time since SCI a positive association with the outcome. The overall model was significant $(\mathrm{F}(14,456), p<0.0001)$, with an adjusted $R^{2}$ of 0.20 . Total number of unmet needs (coeff.(b) $=-1.65 ; p<$ $0.001 ; 95 \%$ CI: $-2.19,-1.10$ ) was likewise negatively related to life satisfaction squared. Non-Swiss citizenship showed a negative and time since SCI onset and traumatic etiology a positive association with the outcome. The 
Table 3 Predictors of expressed service needs

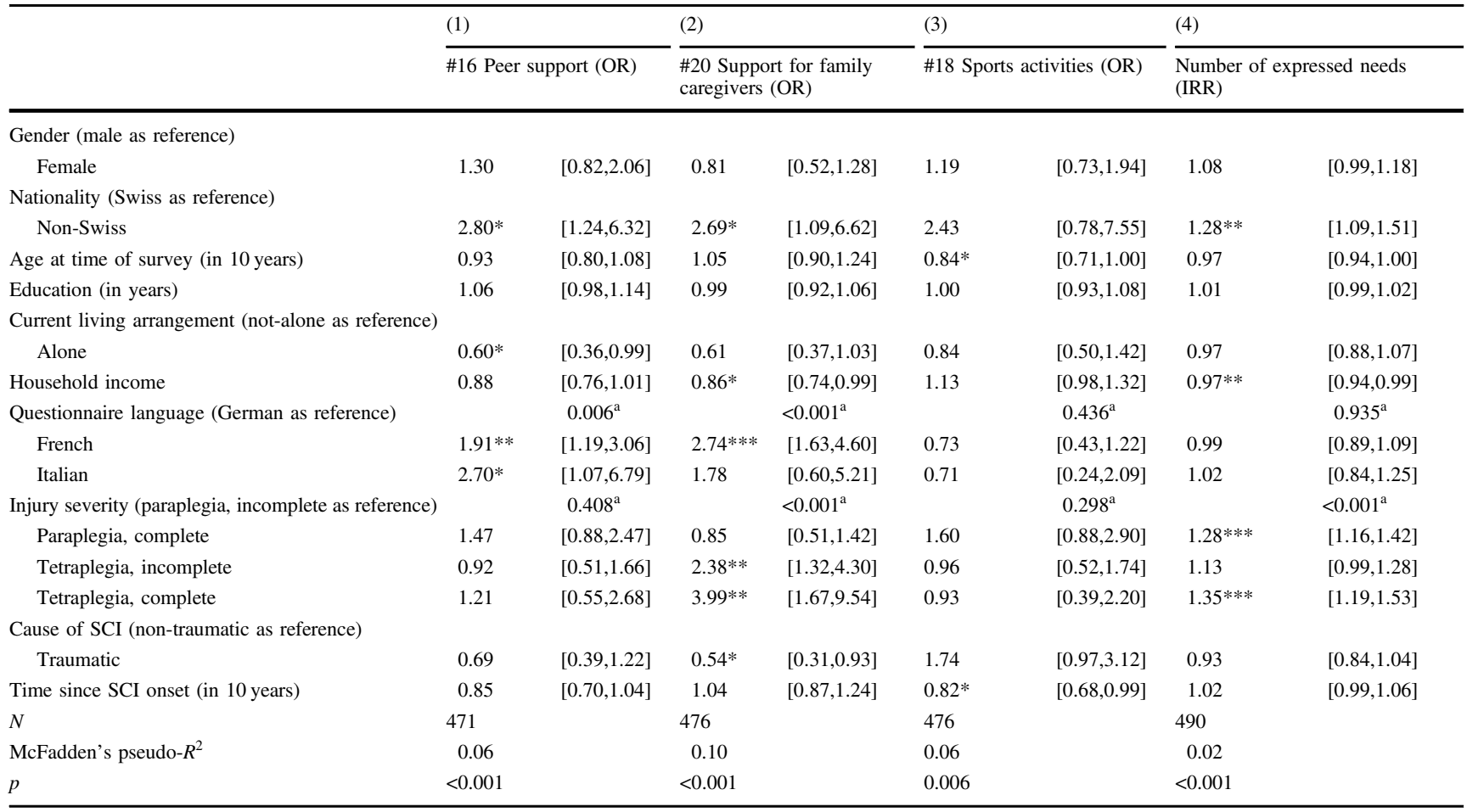

$O R$ odds ratio, IRR incidence rate ratio, $95 \%$ confidence intervals in brackets

${ }^{\mathrm{a}} p$-values derived from global Wald $\chi^{2}$ tests; constants omitted

$* p<0.05, * * p<0.01, * * * p<0.001$

overall model was significant $(\mathrm{F}(14,456), p<0.0001)$, with an adjusted $R^{2}$ of 0.21 .

\section{Discussion}

In this study we examined the prevalence, determinants and consequences of specific as well as cumulative expressed and unmet service needs of persons with SCI living in the community in Switzerland. For the 20 service needs, we found a statistically significant high positive correlation between need prevalence and average level of need fulfillment. Furthermore, there were no clear instances of service needs with simultaneously a very high prevalence and a particularly low average fulfillment level. Taken together, these results may suggest that the existing service provision system had indeed identified and addressed the most important needs in the past. However, less prevalent needs are also more easily overlooked, even as they can still be important to the affected persons.

Furthermore, there clearly remains room for improvement as only two service needs (\#8 general health care and \#1 accessible housing) reached an average fulfillment score of more than 3 ( $=$ to a large extent). Moreover, there were a number of needs, most notably \#16 peer support, that fell below an average score of $2(=$ to some extent) and whose prevalence ranged between $33 \%$ and $55 \%$. In terms of potential predictors of specific and total expressed and unmet service needs, language region, household income, injury severity, gender, and nationality exhibited most frequently statistically significant associations. However, model fit was generally low, pointing to the importance of other explanatory factors not included in the models. With regard to the potential impact of the total number of expressed and the total number of unmet needs, we found a negative adjusted association with life satisfaction.

\section{Prevalence and level of fulfillment of service needs}

The most and the least frequently endorsed service needs identified in our study mirror similar findings of a small-scale [23] and a large-scale [13] survey among persons with SCI living in Canada, a country of comparable socio-economic development status as Switzerland. For instance, among people with a traumatic SCI etiology, $92.6 \%$ indicated a need for equipment and technical aids and $90.0 \%$ for general health care. Conversely, only $31.5 \%$ stated a need for SCI peer support and $33.2 \%$ for job training [13]. 


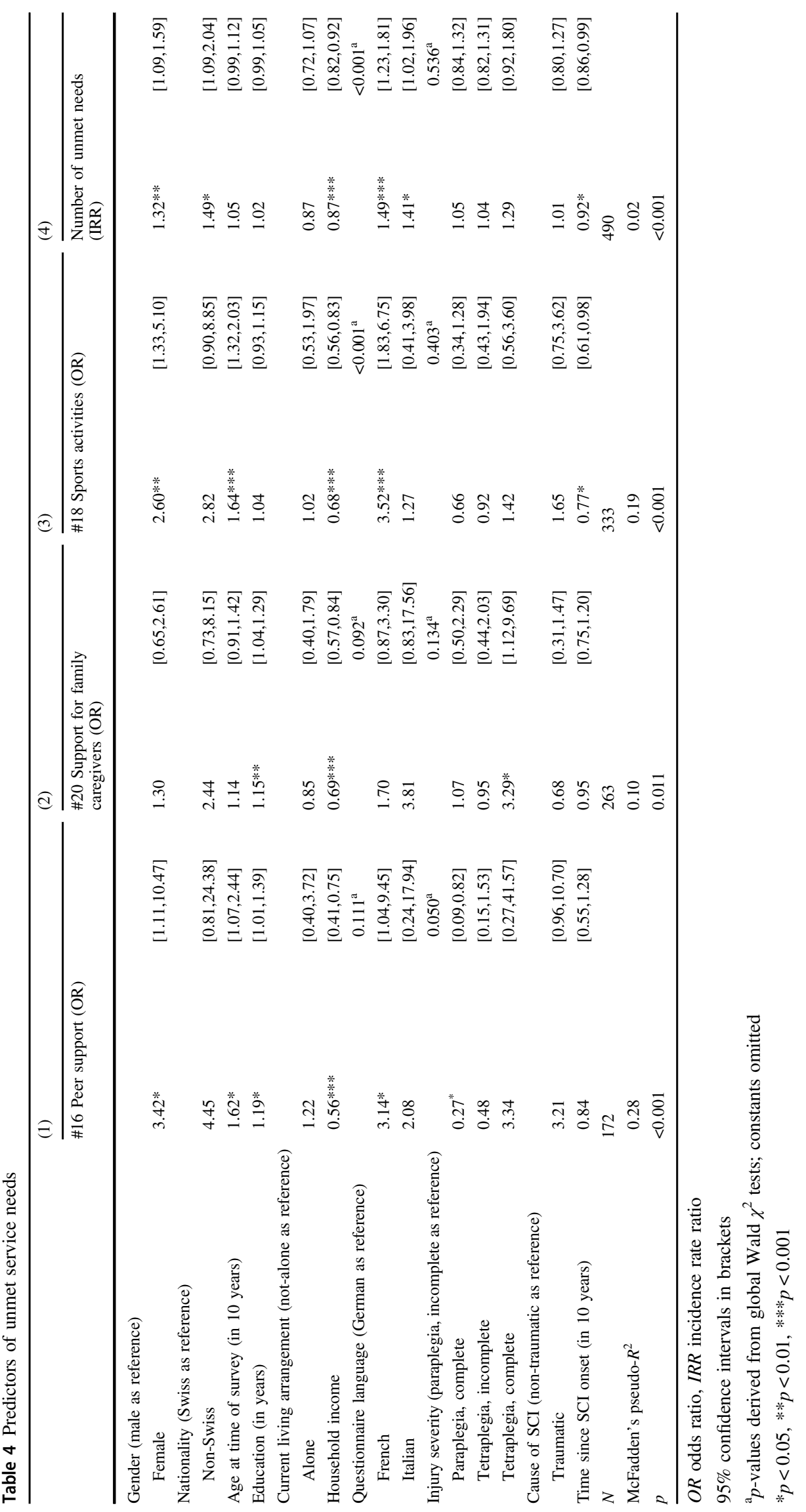




\section{Predictors of expressed and of unmet service needs}

We identified a number of factors associated with expressed and/or unmet needs, albeit with low model fit. More specifically, French language region had a positive association with expressing a need for peer support and for support for family caregivers but did not have a significant effect on unmet needs in these domains. By contrast, French language region increased the likelihood of an unmet need in the area of sports activities and predicted a larger total number of unmet needs. Although these results could merely reflect translation issues or cultural discrepancies in perceived service needs, they might also point to potential regional differences in the provision of services for persons with SCI which may warrant further research. For instance, variations in the health care utilization patterns of persons with SCI had previously been found across 45 Swiss regions [24].

Injury severity was associated with a need for support for family caregivers (with people with tetraplegia being juxtaposed with people with paraplegia) and total number of expressed needs (with complete being juxtaposed with incomplete), but did not show an impact on unmet needs, neither single nor aggregate. This might suggest that although persons with a more severe disability require a broader range of services, the service provision system does cater to these needs in no less a way than the needs of persons with less severe lesions. This result is largely in line with Noreau et al. [12] who found that across their 13 covered domains there were more frequent differences between lesion groups with regard to expressed needs rather than met needs.

Whereas gender was not associated with expressed needs, female respondents were more likely to indicate unmet needs regarding sports activities and peer support and also tended to report a higher total number of unmet needs. This appears at variance with findings by Sweet et al. [25] where in a recursive partitioning analysis only the total number of unmet SCI-related service needs was predictive of a (un)fulfilled need for peer support.

Non-Swiss citizenship predicted a larger and household income a smaller total number of expressed service needs, and the direction of their impact on the three individual expressed needs was consistent but not always significant. Household income, however, showed in addition a reliably significant negative association with the three specific unmet needs as well as with the total number of unmet needs. This is in tune with previous research findings which showed that whereas higher income groups were more likely to report that their needs were met than lower income groups, income showed a less consistent association with expressed needs [12]. These results suggest that despite the comparably comprehensive social security coverage in
Switzerland there remains an economic gradient. They also raise the question of whether eligible persons are sufficiently aware of the availability of the services and direct financial support provided by non-governmental organizations such as the Swiss Paraplegic Foundation.

\section{Impact of cumulative expressed and unmet service needs on life satisfaction}

The established negative association of total number of expressed needs and total number of unmet needs with life satisfaction is consistent with previous research involving both people with SCI $[9,16]$, and people with other health conditions such as traumatic brain injury $[26,27]$ and cancer [28]. More specifically, using a structural equation modeling approach, Sweet et al. [16] found a direct negative effect of the two latent constructs "unmet vital needs" (e.g., general health care; accessible housing) and "unmet developmental needs" (e.g., emotional counseling; peer support) on quality of life defined as satisfaction in various areas of life. However, conceptually and given the crosssectional design of our and the cited studies, we cannot rule out the possibility that it was life satisfaction that exerted an influence on the appraisal of perceived service needs. Furthermore, there might also have been an underlying common cause at work that was not controlled for in the models (e.g., personal factors such as positive/negative mood) and that could account for the (spurious) association.

\section{Limitations}

Apart from the inherent shortcomings of a cross-sectional study design in terms of attribution of causality and the difficulties of generalizing the present results to other countries with different social insurance and service provision systems, two additional limitations need to be highlighted. First, although it seems natural to rely on participants' subjective service needs appraisals in order to identify potential gaps, such self-reported information might be prone to over- and underreporting. For effective planning and delivery of participation-enhancing services for community-dwelling persons with SCI it would be essential to have additional professional assessments of service needs and their fulfillment. Second, the utility of our results is circumscribed by the fact that we did not have information on the perceived quality of and satisfaction with currently provided services.

\section{Conclusions}

Service needs with a high prevalence among communitydwelling persons with SCI living in Switzerland seem to be 
adequately met by the current service provision system. However, rehabilitation professionals should remain alert to clients' specific and cumulative unmet needs, in particular with respect to less common ones, and their impact on successful community reintegration and life satisfaction.

\section{Data archiving}

Owing to our commitment to SwiSCI study participants and their privacy, datasets generated during the current study are not made publicly available. The SwiSCI Study Center requires, on behalf of the SwiSCI Study Group, contact prior to any planned data usage (contact@swisci.ch).

Acknowledgements We are grateful to all the participants of the SwiSCI survey for their time and effort spent in responding to the questions. We further thank the personnel of the SwiSCI study center and the SwiSCI Steering Committee whose members are: Xavier Jordan, Bertrand Léger (Clinique Romande de Réadaptation, Sion); Michael Baumberger, Hans Peter Gmünder (Swiss Paraplegic Center, Nottwil); Armin Curt, Martin Schubert (University Clinic Balgrist, Zürich); Margret Hund-Georgiadis, Kerstin Hug (REHAB Basel, Basel); Thomas Troger (Swiss Paraplegic Association, Nottwil); Daniel Joggi (Swiss Paraplegic Foundation, Nottwil); Hardy Landolt (Representative of persons with SCI, Glarus); Nadja Münzel (Parahelp, Nottwil); Mirjam Brach, Gerold Stucki (Swiss Paraplegic Research, Nottwil); Christine Fekete (SwiSCI Coordination Group at Swiss Paraplegic Research, Nottwil).

Funding The study was financed in the framework of the Swiss Spinal Cord Injury Cohort Study (SwiSCI, www.swisci.ch), supported by the Swiss Paraplegic Foundation.

Author contributions BT, MB, and MP designed the study. BT conducted the data analysis and prepared the article. AG, MB and MP contributed to the data interpretation and article preparation.

\section{Compliance with ethical standards}

Conflict of interest The authors declare that they have no conflict of interest.

Ethical approval The study was approved by the ethics committees of the four Swiss cantons with SCI-specialized centers (Lucerne, Zurich, Basel-Stadt and Valais) [18].

Publisher's note: Springer Nature remains neutral with regard to jurisdictional claims in published maps and institutional affiliations.

\section{References}

1. United Nations. Convention on the rights of persons with disabilities. http://www.ohchr.org/EN/HRBodies/CRPD/Pages/ ConventionRightsPersonsWithDisabilities.aspx. Accessed 20 Jan 2018.

2. WHO. World report on disability. Geneva: World Health Organization; 2011.

3. Lukersmith $\mathrm{S}$ (ed). Guidance on the support needs for adults with spinal cord injury. Sydney: icare (Insurance and Care NSW); 2017.
4. Moreno A, Zidarov D, Raju C, Boruff J, Ahmed S. Integrating the perspectives of individuals with spinal cord injuries, their family caregivers and healthcare professionals from the time of rehabilitation admission to community reintegration: protocol for a scoping study on SCI needs. BMJ Open. 2017; 7.

5. Bickenbach J, Officer A, Shakespeare T, von Groote P (eds). International perspectives on spinal cord injury. Geneva: World Health Organization, International Spinal Cord Society; 2013.

6. Cox RJ, Amsters DI, Pershouse KJ. The need for a multidisciplinary outreach service for people with spinal cord injury living in the community. Clin Rehabil. 2001;15:600-6.

7. Kennedy P, Sherlock O, McClelland M, Short D, Royle J, Wilson C. A multi-centre study of the community needs of people with spinal cord injuries: the first 18 months. Spinal Cord. 2010;48:15-20.

8. Van Loo MA, Post MW, Bloemen JH, van Asbeck FW. Care needs of persons with long-term spinal cord injury living at home in the Netherlands. Spinal Cord. 2010;48:423-8.

9. Post MW, van Asbeck FW, van Dijk AJ, Schrijvers AJ. Services for spinal cord injured: availability and satisfaction. Spinal Cord. 1997;35:109-15.

10. Biering-Sørensen F, Hansen RB, Biering-Sørensen J. Mobility aids and transport possibilities 10-45 years after spinal cord injury. Spinal Cord. 2004;42:699-706.

11. Biering-Sørensen T, Hansen RB, Biering-Sørensen F. Home aids and personal assistance 10-45 years after spinal cord injury. Spinal Cord. 2009;47:405-12.

12. Noreau L, Noonan VK, Cobb J, Leblond J, Dumont FS. Spinal cord injury community survey: understanding the needs of Canadians with SCI. Top Spinal Cord Inj Rehabil. 2014; 20:265-76.

13. Noreau L, Noonan VK, Cobb J, Leblond J, Dumont FS. Spinal cord injury community survey: a national, comprehensive study to portray the lives of Canadians with spinal cord injury. Top Spinal Cord Inj Rehabil. 2014;20:249-64.

14. Hertig-Godeschalk A, Gemperli A, Arnet U, Hinrichs T. Availability and need of home adaptations for personal mobility among individuals with spinal cord injury. J Spinal Cord Med. 2018;41:91-101.

15. Donnelly C, McColl MA, Charlifue S, Glass C, O’Brien P, Savic $\mathrm{G}$, et al. Utilization, access and satisfaction with primary care among people with spinal cord injuries: a comparison of three countries. Spinal Cord. 2007;45:25-36.

16. Sweet SN, Noreau L, Leblond J, Dumont FS. Understanding quality of life in adults with spinal cord injury via SCI-related needs and secondary complications. Top Spinal Cord Inj Rehabil. 2014;20:321-8.

17. Kennedy P, Lude P, Taylor N. Quality of life, social participation, appraisals and coping post spinal cord injury: a review of four community samples. Spinal Cord. 2006; 44:95-105.

18. Brinkhof MW, Fekete C, Chamberlain JD, Post MW, Gemperli A. Swiss national community survey on functioning after spinal cord injury: Protocol, characteristics of participants and determinants of non-response. J Rehabil Med. 2016;48: 120-30.

19. FORS. Swiss Household Panel, wave 14 (individual questionnaire). Lausanne: FORS; 2012.

20. Brown LD, Cai TT, DasGupta A. Interval estimation for a binomial proportion. Stat Sci. 2001;16:101-33.

21. Hodgetts S, Zwaigenbaum L, Nicholas D. Profile and predictors of service needs for families of children with autism spectrum disorders. Autism. 2015;19:673-83.

22. Stekhoven DJ, Bühlmann P. MissForest-non-parametric missing value imputation for mixed-type data. Bioinformatics. 2012;28:112-8. 
23. Beauregard L, Guindon A, Noreau L, Lefebvre H, Boucher N. Community needs of people living with spinal cord injury and their family. Top Spinal Cord Inj Rehabil. 2012;18:122-5.

24. Ronca E, Scheel-Sailer A, Koch HG, Gemperli A. Health care utilization in persons with spinal cord injury: part 2-determinants, geographic variation and comparison with the general population. Spinal Cord. 2017;55:828.

25. Sweet SN, Noreau L, Leblond J, Martin Ginis KA. Peer support need fulfillment among adults with spinal cord injury: relationships with participation, life satisfaction and individual characteristics. Disabil Rehabil. 2016;38:558-65.
26. Heinemann AW, Sokol K, Garvin L, Bode RK. Measuring unmet needs and services among persons with traumatic brain injury. Arch Phys Med Rehabil. 2002;83:1052-9.

27. Schulz-Heik RJ, Poole JH, Dahdah MN, Sullivan C, Adamson MM, Date ES, et al. Service needs and barriers to care five or more years after moderate to severe TBI among Veterans. Brain Inj. 2017;31:1287-93.

28. Hansen DG, Larsen PV, Holm LV, Rottmann N, Bergholdt SH, Søndergaard J. Association between unmet needs and quality of life of cancer patients: a population-based study. Acta Oncol. 2013;52:391-9. 